\title{
Molecular Mechanism of Facilitated Dissociation of Fis Protein from DNA
}

\author{
Min-Yeh Tsai, ${ }^{\dagger}$ Bin Zhang, Weihua Zheng, ${ }^{\dagger}$ and Peter G. Wolynes*, ${ }^{\dagger}$ \\ ${ }^{\dagger}$ Department of Chemistry, and Center for Theoretical Biological Physics, Rice University, Houston, Texas 77005, United States \\ ${ }^{\ddagger}$ Department of Chemistry, Massachusetts Institute of Technology, Cambridge, Massachusetts 02139, United States
}

\section{Supporting Information}

ABSTRACT: Fis protein is a nucleoid-associated protein that plays many roles in transcriptional regulation and DNA site-specific recombination. In contrast to the naive expectation based on stoichiometry, recent single-molecule studies have shown that the dissociation of Fis protein from DNA is accelerated by increasing the concentration of the Fis protein. Because the detailed molecular mechanism of facilitated dissociation is still not clear, in this study, we employ computational methods to explore the binding landscapes of Fis:DNA complexes with various stoichiometries. When two Fis molecules are present, simulations uncover a ternary complex, where the originally bound Fis protein is partially dissociated from DNA. The simulations support a three-state sequential kinetic model $(N \rightleftarrows I \rightarrow D)$ for facilitated dissociation, thus explaining the concentration-dependent dissociation.

$\mathrm{T}$ he expression of genes is modulated at multiple levels of complexity so as to maintain life. An orchestrated set of protein-protein and protein-DNA interactions initiates the reading and encoding of genetic information. In this interacting network one protein product very often regulates the translation into protein of other genes by enhancing or suppressing their transcription specifically. Fis (Factor for inversion stimulation) protein is an example of this kind of regulator. First found in Escherichia coli as a nucleoid-associated protein, Fis protein plays a role in stimulating DNA inversion during site-specific DNA recombination. ${ }^{1-3}$ Later, it was found that Fis protein also activates transcription of several physiologically related genes by interacting with the $\alpha$-CTD subunit of RNA polymerase on promoters.

Although the relevance of Fis binding to DNA for regulating a range of genes is widely acknowledged, ${ }^{5}$ how the Fis protein dissociates from DNA once it is no longer needed is still not clear. Recent experimental studies using single-molecule techniques show that other protein molecules facilitate the removal and renewal of Fis proteins that are bound to DNA. ${ }^{6}$ When the concentration of Fis protein itself increases, the dissociation of already bound Fis protein increases too while all its kinetics exhibits a single exponential decay. ${ }^{6}$ Dissociation can be facilitated not only by the Fis molecule itself but also by the action of the bacterial protein $\mathrm{HU}$, the yeast HMGB protein NHP6A, ${ }^{6}$ as well as other DNA segments. ${ }^{7}$ Facilitated dissociation has been observed experimentally for a wide variety of genetic systems, including DNA polymerase exchange in phage T7 replisome during DNA replication, ${ }^{8}$ dissociation of metal-dependent transcription factor CueR by apo-CueR, ${ }^{9}$ and the facilitated dissociation of eukaryotic High-Mobility Group B (HMGB) protein by itself. ${ }^{10}$ In addition, facilitated dissociation has also be seen in single-stranded DNA binding, ${ }^{11}$ highlighting its widespread importance across all types of regulatory processes in cells. From the kinetic point of view (but not as we shall see the mechanistic point of view), molecular stripping in the $\mathrm{NF} \kappa \mathrm{B} / \mathrm{I} \kappa \mathrm{B} / \mathrm{DNA}$ system ${ }^{12}$ appears analogous: the biology of regulation does not always have the patience to wait for spontaneous dissociation to occur!

Obviously, all examples of facilitated dissociation must require a termolecular sequence of encounters (the interaction between one protein in solution and its partner) that at some point leads to the formation of a transient ternary complex. ${ }^{6,7,9,11,13}$ In this study, we show that a three-state sequential kinetic reaction suffices to understand the concentration dependence and single exponential kinetics observed from experiment. ${ }^{6,14}$ Theoretical analysis and simulations capture the structure and energies of ternary complexes so as to explain the experimental concentrationdependent protein exchange. In the Fis system, a key role is played by the multiple domain character of the protein which allows partial dissociation of the Fis protein from the DNA when a partner approaches. ${ }^{13}$

To understand in molecular detail how the Fis protein is released from DNA spontaneously and how this dissociation is assisted by other Fis proteins, we have computationally explored the binding landscapes of protein dissociation from DNA using a state-of-the-art coarse-grained protein/DNA model (AWSEM/3SPN.2C) (see Supporting Information), which we have developed. This model has been previously applied to study molecular stripping of $\mathrm{NF} \kappa \mathrm{B}$ from DNA by $\mathrm{I} \kappa \mathrm{B}^{12}$ and the disassembly of the nucleosome. ${ }^{15}$ Although Fis protein does exhibit sequence specific binding, its preferred 15 base pair core recognition signal is poorly conserved and thus its sequence specificity is weak. ${ }^{4,16,17}$ The major part of the binding of Fis protein to DNA in the simulation comes from nonspecific electrostatic interactions which can be modeled using Debye-Hückel potentials. We compare the two different simulations scenarios, one for spontaneous dissociation and the other for facilitated dissociation using free energy profile analysis. These simulations uncover a partially dissociated Fis conformation on DNA in which only a single domain remains

Received: August 11, 2016

Published: September 29, 2016 
(a)

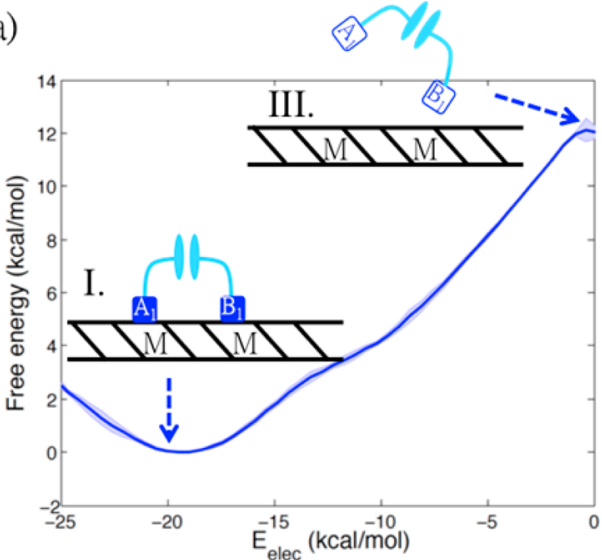

(c) Binary complex (Fis 1:DNA)

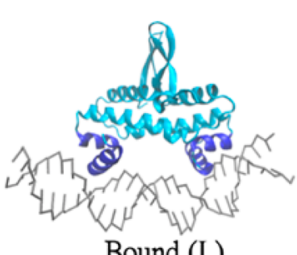

Bound (I.)

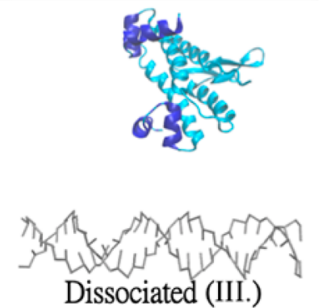

(b)

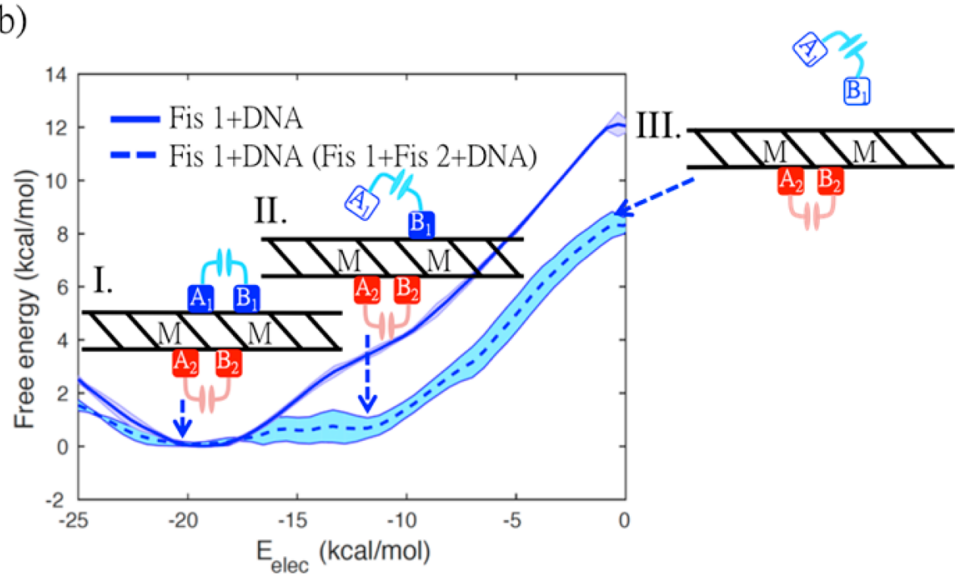

(d)

Ternary complex (Fis 1:Fis 2:DNA)

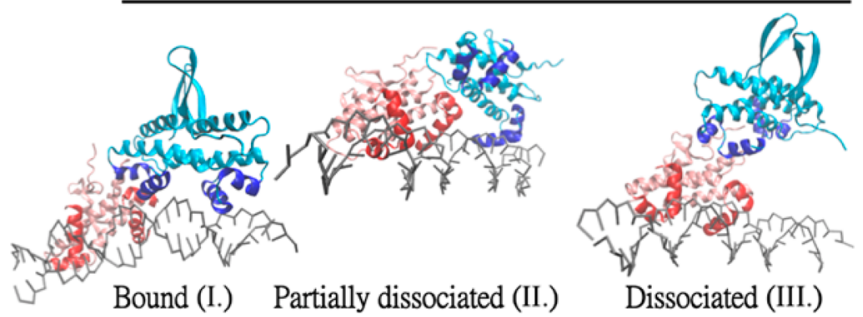

Figure 1. Free energy profile as a function of electrostatic energy $E_{\text {elec }}$ of the interaction of the protein Fis 1 (colored in blue) with DNA is shown. (a) The profile for spontaneous dissociation of Fis 1:DNA complex. (b) The profile for the spontaneous dissociation of Fis 1:DNA complex (solid line) and its facilitated dissociation (dashed line) due to the additional Fis 2 protein are compared in the same plot. The shaded area indicates error bars representing the standard deviation of the mean. Example configurations of the Fis 1:Fis 2:DNA ternary complex at various values of the interaction $E_{\text {elec }}$ are shown in the plot. The arrows indicate the bound, intermediate, and dissociated states. Cartoon diagram is used to visually guide the reader through the configurations of the ternary complex. The letter " $\mathrm{M}$ " refers to the major grooves of the DNA while the neighboring empty space denotes the DNA minor grooves. Configurations of different binary (c) and ternary (d) complexes are shown; they are labeled with numbers shown in panels a and $b$, respectively.

bound to the DNA. We show that the detailed dissociation mechanism relies upon the frustration of the electrostatic interactions of the two distinct DNA-binding domains (anchors) of the Fis protein in its interactions with the DNA. We postulate the strategy used here can be further extended to investigate also heterologous dissociation facilitated by proteins of different kinds ${ }^{6}$ as well as the facilitated dissociation at one genetic site by other DNA segments ${ }^{7}$ that can couple gene regulation to large scale genome 3D structure.

These phenomena are made evident by computing free energy profiles using the electrostatic interaction energy between an individual Fis protein (Fis 1, blue) and DNA as a progress coordinate to follow the dissociation both for the complex of a single Fis with DNA and for the DNA complex with a pair of Fis proteins, mimicking spontaneous and facilitated dissociation, respectively. These profiles are shown in Figure. 1. For spontaneous dissociation (solid line), the free energy profile shows that the Fis 1:DNA binding landscape is clearly funneled, having only one free energy basin at $E_{\text {elec }} \sim$ $-20 \mathrm{kcal} / \mathrm{mol}$, which corresponds to the normal bound state complex. The free energy barrier for dissociation is about 12 $\left(\mathrm{kcal} / \mathrm{mol}\right.$ ), consistent both with experiment ${ }^{18}$ (see Supporting Information) and a previous theoretical estimation. ${ }^{19}$ Interestingly, at $\sim-12 \mathrm{kcal} / \mathrm{mol}$, a free energy plateau is vaguely recognized, signaling the presence of an intermediate state. This intermediate state is clearly observed in the free energy basin for facilitated dissociation (dashed line). In the structures in this basin the first Fis has been partially dissociated from the DNA.
Such a partially dissociated structure is not thermodynamically stable in the absence of the second Fis molecule. Also, we see from the profile that the dissociation barrier decreases from $\sim 12$ to $\sim 9 \mathrm{kcal} / \mathrm{mol}$ when a second Fis is present. This decrease is consistent with the increased dissociation rate that is experimentally observed at increased protein concentration. ${ }^{6}$ The configurations explored within this new free energy basin correspond to an ensemble of configurations of the ternary Fis 1:Fis 2:DNA complex. Overall, the perturbed binding landscape becomes rugged and less funneled.

The nature of the intermediate state becomes even more apparent when the free energy profile is projected onto several order parameters simultaneously. In particular, we use the interaction of both individual chains of Fis 1 (Fis protein is a dimer), chain $\mathrm{A}$ and chain $\mathrm{B}$, with the DNA as simultaneous progress coordinates. The resulting two-dimensional free energy surfaces are shown in Figure 2. Both the spontaneous dissociation (a) and facilitated dissociation (b) exhibit an obvious basin at $(-10,-10)$, which corresponds to the free energy minima at $E_{\text {elec }} \sim-20 \mathrm{kcal} / \mathrm{mol}$ seen in Figure 1. Basins along the off-diagonals $(-10,0)$ and $(0,-10)$ are in harmony with the idea that the intermediate state observed in Figure 1 corresponds to the partial dissociation of Fis 1 with one of its monomers (anchors) dissociating from the DNA. Example configurations from these basins are shown in Figure 1d. The partially dissociated state of Fis 1 is stabilized by the presence of Fis 2 as both proteins compete for binding with the DNA (see Figure S7 in Supporting Information). In the naive case, if the 
(a)

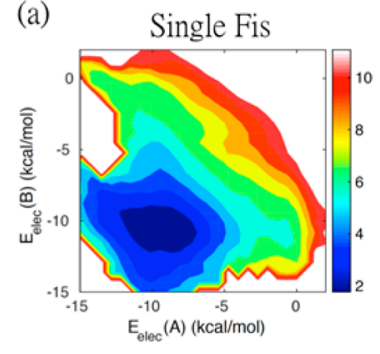

(b)

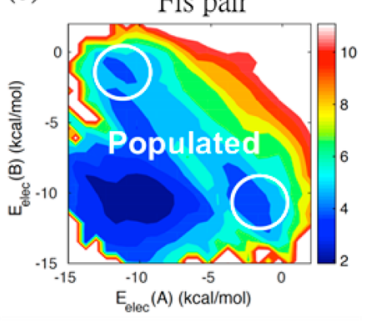

(c)

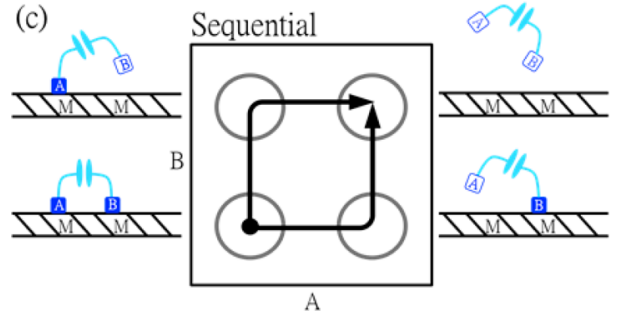

Figure 2. Mechanism of dissociation of Fis protein from DNA is explored using a two-dimensional free energy surface as a function of the electrostatic interaction energies of the individual monomeric domains, chain A and B, from Fis 1 with the DNA. (a) Spontaneous dissociation. (b) Facilitated dissociation. (c) Schematic diagram showing that the Fis protein dissociates through two-step parallel sequential mechanisms. Cartoon representation is used to illustrate the configuration of the Fis:DNA complex.

DNA has only two binding sites, both of them will be occupied by Fis 1 in the bound state with $E_{\text {elec }} \sim-20 \mathrm{kcal} / \mathrm{mol}$. Introducing Fis 2 favors the intermediate state, as Fis 2 interacts with the newly exposed binding site from the Fis 1's partial dissociation. On the other hand, a symmetric pattern of new free energy minima in Figure $2 b$ appears showing that the dissociation of Fis protein from DNA can follow two symmetric parallel sequential mechanisms, illustrated in Figure 2c.

The free energy profiles computed for the facilitated dissociation allow us to formulate the three-state kinetic scheme shown in Figure 3. The three states in the kinetic

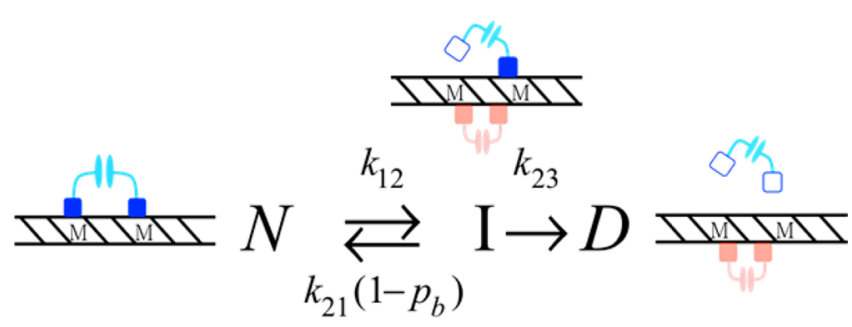

Figure 3. Three-state kinetic model is shown. $N$, $I$, and $D$ represent the bound state, the intermediate state, and the dissociated state of a single Fis:DNA complex, respectively. Note that $I$ refers to the partially dissociated ensemble where a DNA binding site is available for the binding of an additional Fis protein (in transparent pink). The binding probability is denoted by $p_{\mathrm{b}}$.

scheme, $N, I, D$, represent three different conformational ensembles of the Fis:DNA complex. $N$ refers to the fully bound ensemble (with both two Fis anchors well bound onto the DNA); I denotes the partially dissociated state (having only one Fis anchor bound); and $D$ represents the dissociated state (where the Fis protein is completely detached from DNA, so there are no bound Fis anchors). The concentration dependence of the measured dissociation rate comes about from a population shift between the $N$ and $I$ ensembles, due to the competition for a common binding site. In the $I$ state one of the binding sites is exposed, allowing other solution-phase proteins to have access to the DNA (represented by an additional transparent Fis protein in pink shown in Figure 3). $p_{\mathrm{b}}$ refers to the probability of the binding site being occupied via solutionphase proteins (Fis 2 in our simulation). This binding event is responsible for the change in the reverse rate constant, scaled by $1-p_{\mathrm{b}}$. Both the exact solution of the kinetics of the threestate model and a time scale separation analysis are provided in the Supporting Information. This analysis shows the experimentally observed single-exponential kinetics corresponds to the slowest relaxation of all characteristic time scales.

The experimentally measured phenomenological rates $k_{0}$ and $k_{1}$ (dissociation rate $k_{\text {off }}=k_{0}+k_{1} c$ ) are given in terms of the time constants shown in Figure 3 as

$$
k_{0}=\frac{k_{12} k_{23}}{k_{12}+k_{21}}
$$

and

$$
k_{1}=\frac{k_{12} k_{23} \cdot k_{21}}{\left(k_{12}+k_{21}\right)^{2}} c_{0}^{-1} \mathrm{e}^{-\beta \Delta \varepsilon}
$$

where $\Delta \varepsilon=\varepsilon-\mu_{0}, \varepsilon$ is the binding free energy of a single Fis protein on DNA and $\mu_{0}$ is the chemical potential of Fis protein in solution at a reference concentration $c_{0}$. $\Delta \varepsilon$ therefore indicates the free energy difference upon taking the protein from solution and placing it on the DNA binding sites, which is predicted to be $\sim 9 \mathrm{kcal} / \mathrm{mol}$ from the free energy profile of facilitated dissociation (see Figure $1 \mathrm{~b}$ ). Similarly, $k_{\mathrm{ij}}$ can also be estimated from the free energy profile using Kramers theory. These parameters, all ab initio obtained from our simulations, yielded $k_{0}=5.3 \times 10^{-3}\left(\mathrm{~s}^{-1}\right)$, which is in reasonable agreement with the experimental value $1 \times 10^{-3}\left(\mathrm{~s}^{-1}\right)$. The diffusion coefficient in the kinetic prefactor was calculated along multiple coordinates in relevant diffusion regime (see Supporting Information).

To clarify the relative importance of protein-protein and protein-DNA interactions in the intermediate, we also monitored the distance between a given Fis protein and the other Fis protein (or DNA) in the simulation trajectories (see Figure S8). The results show that facilitated dissociation is caused largely by protein-DNA interaction. A particular conformation of Fis:DNA complex is selected prior to dissociation (see Figure S9) so that facilitated dissociation occurs by conformational selection in the original Fis:DNA complex (see Supporting Information).

Finally, we have carried out a frustration analysis. Frustration is often a signal of an evolved need for bistability. ${ }^{20}$ Frustration is a measure that quantifies conflicting interactions in spatially localized regions in proteins, through the $Z$ score of the native energy compared with the energy of a set of decoys. ${ }^{21}$ We have noted that electrostatics leads to frustration in the DNAbinding regions (the two anchor parts) of Fis protein. ${ }^{22}$ To explore the role of their frustration in facilitated dissociation, we can simultaneously monitor the degree of frustration on the Fis proteins' DNA-binding domains (anchors) during dissociation. The frustrated interactions of individual anchors are relieved upon binding to DNA so that they become minimally frustrated. The frustration profile localizes protein-DNA interactions (see Figure S11) that identify facilitated dissociation (see Supporting Information). 


\section{ASSOCIATED CONTENT}

\section{S Supporting Information}

The Supporting Information is available free of charge on the ACS Publications website at DOI: 10.1021/jacs.6b08416.

Simulation methods; further analysis (PDF)

\section{AUTHOR INFORMATION}

Corresponding Author

*pwolynes@rice.edu

Notes

The authors declare no competing financial interest.

\section{ACKNOWLEDGMENTS}

The project described was funded by the NSF sponsored Center for Theoretical Biological Physics (Grants PHY1308264 and PHY-1427654) with additional support from NIH Grant R01 GM44557. Support from the National Institute of General Medical Sciences PPG Grant P01 GM071862 and from the D. R. Bullard-Welch Chair (Grant C-0016) at Rice University to P.G.W. is greatly appreciated.

\section{REFERENCES}

(1) Johnson, R. C. Microbiol. Spectrum 2015, 3, 1-36.

(2) Dhar, G.; Heiss, J. K.; Johnson, R. C. Mol. Cell 2009, 34, 746759.

(3) Heichman, K. A.; Johnson, R. C. Science 1990, 249, 511-517.

(4) Cheng, Y. S.; Yang, W. Z.; Johnson, R. C.; Yuan, H. S. J. Mol. Biol. 2000, 302, 1139-1151.

(5) Bradley, M. D.; Beach, M. B.; de Koning, A. P. J.; Pratt, T. S.; Osuna, R. Microbiology 2007, 153, 2922-2940.

(6) Graham, J. S.; Johnson, R. C.; Marko, J. F. Nucleic Acids Res. 2011, 39, 2249-2259.

(7) Giuntoli, R. D.; Linzer, N. B.; Banigan, E. J.; Sing, C. E.; de la Cruz, M. O.; Graham, J. S.; Johnson, R. C.; Marko, J. F. J. Mol. Biol. 2015, 427, 3123-3136.

(8) Loparo, J. J.; Kulczyk, A. W.; Richardson, C. C.; van Oijen, A. M. Proc. Natl. Acad. Sci. U. S. A. 2011, 108, 3584-3589.

(9) Joshi, C. P.; Panda, D.; Martell, D. J.; Andoy, N. M.; Chen, T.-Y.; Gaballa, A.; Helmann, J. D.; Chen, P. Proc. Natl. Acad. Sci. U. S. A. 2012, 109, 15121-15126.

(10) McCauley, M. J.; Rueter, E. M.; Rouzina, I.; Maher, L. J.; Williams, M. C. Nucleic Acids Res. 2013, 41, 167-181.

(11) Gibb, B.; Ye, L. F.; Gergoudis, S. C.; Kwon, Y.; Niu, H.; Sung, P.; Greene, E. C. PLoS One 2014, 9, e87922.

(12) Potoyan, D. A.; Zheng, W.; Komives, E. A.; Wolynes, P. G. Proc. Natl. Acad. Sci. U. S. A. 2016, 113, 110-115.

(13) Sing, C. E.; de la Cruz, M. O.; Marko, J. F. Nucleic Acids Res. 2014, 42, 3783-3791.

(14) Marko, J. F. Phys. A 2015, 418, 126-153.

(15) Zhang, B.; Zheng, W. H.; Papoian, G. A.; Wolynes, P. G. J. Am. Chem. Soc. 2016, 138, 8126-8133.

(16) Saier, M. H., Jr. Crit. Rev. Biochem. Mol. Biol. 2008, 43, 89-134.

(17) Shao, Y.; Feldman-Cohen, L. S.; Osuna, R. J. Mol. Biol. 2008, 376, 771-785.

(18) Skoko, D.; Yoo, D.; Bai, H.; Schnurr, B.; Yan, J.; McLeod, S. M.; Marko, J. F.; Johnson, R. C. J. Mol. Biol. 2006, 364, 777-798.

(19) Cocco, S.; Marko, J. F.; Monasson, R. Phys. Rev. Lett. 2014, 112, 238101.

(20) Ferreiro, D. U.; Komives, E. A.; Wolynes, P. G. Q. Rev. Biophys. 2014, 47, 285-363.

(21) Parra, R. G.; Schafer, N. P.; Radusky, L. G.; Tsai, M. Y.; Guzovsky, A. B.; Wolynes, P. G.; Ferreiro, D. U. Nucleic Acids Res. 2016, 44, W356-W360.

(22) Tsai, M.-Y.; Zheng, W.; Balamurugan, D.; Schafer, N. P.; Kim, B. L.; Cheung, M. S.; Wolynes, P. G. Protein Sci. 2016, 25, 255-269. 